\title{
Fault Diagnosis for Power System Using Time Sequence Fuzzy Petri Net
}

\author{
Sun Yan Bin ${ }^{1, \text { a }}$, Chen Yi Ping ${ }^{1, a}$, Bai Zhan ${ }^{2, b}$, Miao Shi Hong ${ }^{2, b}$, Yao Wei ${ }^{2, b}$, \\ Wen Jinyu ${ }^{2, b}$, Hou Yun $\mathrm{He}^{3, \mathrm{c}}$ \\ ${ }^{1}$ China Southern Power Grid Company, Guangzhou, China \\ ${ }^{2}$ School of Electrical and Electronic Engineering, Huazhong University of Science and Technology, \\ Wuhan, China \\ ${ }^{3}$ Department of Electrical and Electronic Engineering, The University of Hong Kong, Hong Kong \\ asunyb@csg.cn, ${ }^{a}$ chenyiping@csg.cn, ${ }^{b}$ hphdbai@163.com, bshmiao@hust.edu.cn, \\ bw.yao@hust.edu.cn, bjinyu.wen@hust.edu.cn, cyhhou@eee.hku.hk
}

Keywords: Fault diagnosis, power system, time sequence, fuzzy petri net.

\begin{abstract}
In this paper, time sequence fuzzy petri net (TSFPN) is proposed to build power system fault diagnosis models. Firstly, on the basic of taking the influence of incomplete and uncertain relay protection alarm information on fault diagnosis into consideration, the structure of TSFPN and the fuzzy inference approach are proposed. Then, the time constraint characteristics of alarm information and the time sequence checking method are introduced. Finally, by several simulation examples, the fault tolerance and validity of the proposed method is testified, even in the situation with complex fault scenario.
\end{abstract}

\section{Introduction}

The fault diagnosis of power system is a process of identifying component failures by analyzing alarm information. At present, a series of artificial intelligence analysis methods have been applied to fault diagnosis field, such as expert system [1], artificial neural network [2], optimization algorithms [3], Bayesian networks [4], petri net [5], etc. In these methods, petri net has strong modeling ability for discrete event systems and can analyze the various processes of events accurately. Thus, petri net possesses abundant application values and has become a research hotspot.

However, in the face of uncertain alarm information of protective relays and circuit breakers, the diagnosis results of traditional petri net are often difficult to meet the requirements. As a result, the concept of fuzzy petri net is put forward. In [6], the authors use fuzzy petri net to build fault diagnosis models, and the malfunction components are judged according to the values of the component places. In [7], the authors propose a method based on directional weighted fuzzy petri net, which builds models in every possible fault spread direction of bus or line. The weights of transition input arcs and output arcs are modified in [8]. In [9], incidence matrix is used to describe time sequence characteristics of alarm information. In [10], a time constraint checking method is introduced, and the results show that the method can improve the accuracy of fault diagnosis in some degree.

Under the above background, a power system fault diagnosis method based on time sequence fuzzy petri net (TSFPN) is proposed in this paper. The adverse influence of incomplete and uncertain alarm information on fault diagnosis is effectively relieved by the establishment of TSFPN model. In addition, through making full use of the time constraints of protective relays and circuit breakers, inconsistent time sequence information can be removed. At last, the fault tolerance and validity of proposed method is testified by several simulation examples.

\section{Basic Concepts of TSFPN}

Definition. TSFPN can be defined by a 6-tuple set:

$$
\mathrm{TSFPN}=\left\{P, T, \boldsymbol{I}, \boldsymbol{O}, \boldsymbol{\theta}, \boldsymbol{T}_{\mathrm{t}}\right\}
$$


Where $P=\left\{p_{1}, p_{2}, \ldots, p_{n}\right\}$ is a finite set of places, and $n$ is the number of places; $T=\left\{t_{1}, t_{2}, \ldots, t_{m}\right\}$ is a finite set of transitions, and $m$ is the number of transitions; $I: P \rightarrow T$ is a mapping from places to transitions, $\boldsymbol{I}=\left[\omega_{i j}\right]$, if there is a directed arc from $p_{i}$ to $t_{j}, \omega_{i j}$ is the weight of the arc, otherwise $\omega_{i j}=0$; $\boldsymbol{O}: T \rightarrow P$ is a mapping from transitions to places, $\boldsymbol{O}=\left[\mu_{i j}\right]$, if there is a directed arc from $t_{i}$ to $p_{j}, \mu_{i j}$ is the confidence coefficient of the arc, otherwise $\mu_{i j}=0 ; \boldsymbol{\theta}=\left[\theta_{1}, \theta_{2}, \ldots, \theta_{n}\right]$ is the confidence coefficient matrix of places, $\theta_{i}$ is the probability that the action state of place $p_{i}$ is true; $\boldsymbol{T}_{\mathrm{t}}=\left\{T_{1}, T_{2}, \ldots, T_{k}\right\}$ is an operation time set of alarm information, and $k$ is the number of alarm information.

Diagrams. Simple power system is shown in Fig.1, which includes 4 buses $\left(\mathrm{B}_{1}-\mathrm{B}_{4}\right), 3$ lines $\left(\mathrm{L}_{1}-\mathrm{L}_{3}\right)$ and 6 circuit breakers $\left(\mathrm{CB}_{1}-\mathrm{CB}_{6}\right)$.

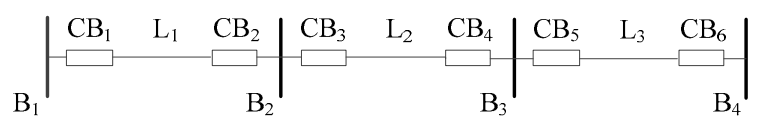

Fig.1 Simple power system

According to the principles of relay protection devices and basic rules of fuzzy inference [6], the TSFPN model of bus $\mathrm{B}_{2}$ is designed and shown in Fig.2, where $p_{1}$ and $p_{2}$ are middle places, $\mathrm{R}$ is the receive end of line, $\mathrm{S}$ is the send end of line, $\mathrm{m}$ is the main protection and $\mathrm{s}$ is the secondary backup protection.

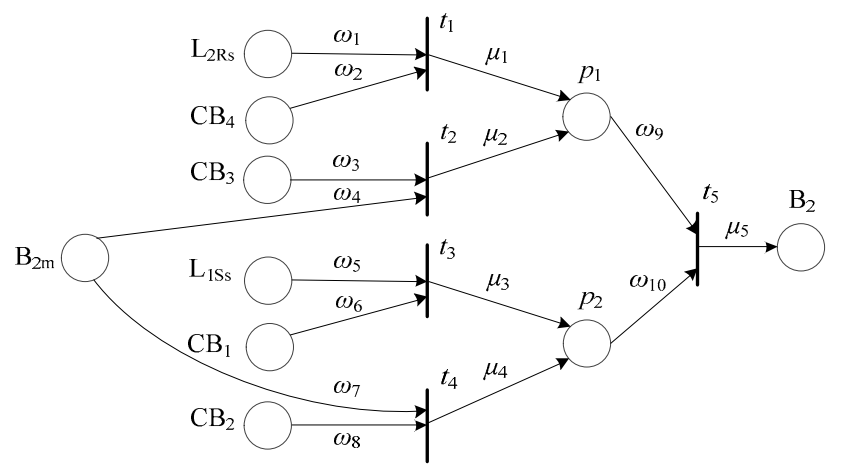

Fig.2 Structure diagram of TSFPN

The structure diagram of TSFPN proposed in this paper contains a combined fuzzy reasoning rule and gives full consideration to the uncertainty of alarm information. In addition, the scale of petri net can be reduced effectively and the calculation speed can be improved.

Inference analysis. In order to make the diagnosis process more clear, the inference of TSFPN adopts the way of matrix manipulation [11]. Let $\boldsymbol{A}$ is $(q \times l)$-dimensional matrix, $\boldsymbol{B}$ is $(l \times h)$ -dimensional matrix and $\boldsymbol{C}$ is $(q \times h)$-dimensional matrix.

1) Matrix multiplication $\bullet$, if $\boldsymbol{C}=\boldsymbol{A} \bullet \boldsymbol{B}, c_{i j}=a_{i 1} b_{1 j}+a_{i 2} b_{2 j}+\ldots+a_{i l} b_{l j}$;

2) Multiplication operator $\otimes$, if $\boldsymbol{C}=\boldsymbol{A} \otimes \boldsymbol{B}, c_{i j}=\max _{1 \leq \gamma \leq l}\left(a_{i j} b_{\gamma j}\right)$.

According to the fuzzy rules and operators, the reasoning process of TSFPN is shown as follows.

\section{$\boldsymbol{E}^{k+1}=\boldsymbol{\theta}^{k} \bullet \boldsymbol{I}$.}

Where $\boldsymbol{E}^{k+1}$ is $m$-dimensional matrix which the element is input probability of transition, $k$ is the number of inference and the initial value is 0 .

$$
\boldsymbol{\theta}^{k+1}=\boldsymbol{E}^{k+1} \otimes \boldsymbol{O}
$$

Where $\boldsymbol{\theta}^{k+1}$ is the confidence coefficient matrix of places after the inference. If only one element in $\boldsymbol{\theta}^{k+1}$ is not zero, the inference is over, otherwise let $k=k+1$ and calculate $\boldsymbol{\theta}^{k+1}$ again.

\section{Time characteristics of alarm information}

Time constraints. Although power system relay protection devices have fixed setting time, the actual operation time of protective relays and circuit breakers cannot be determined accurately. The time constraint characteristics of alarm message are shown in Fig.3. 


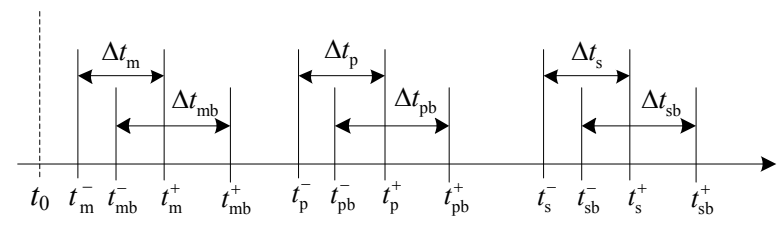

Fig.3 Time constraint characteristics

In Fig. $3, t_{0}$ is the time when fault happens, $\Delta t_{\mathrm{m}}, \Delta t_{\mathrm{p}}$ and $\Delta t_{\mathrm{s}}$ are the time constraint intervals of the main protection, primary backup protection and secondary backup protection, $\Delta t_{\mathrm{mb}}, \Delta t_{\mathrm{pb}}$ and $\Delta t_{\mathrm{sb}}$ are the time constraint intervals of the circuit breakers respectively related to main protection, primary backup protection and secondary backup protection, $t_{m}^{-}$and $t_{m}^{+}$are the minimum and maximum operation time of main protection.

According to the literatures $[11,12]$, from the moment of component faults, define the delay intervals of main protection, circuit breaker related to main protection, primary backup protection, circuit breaker related to primary backup protection, secondary backup protection and circuit breaker related to secondary backup protection are $[10,40],[30,80],[300,500],[320,540],[600,1100]$ and $[620,1140]$, where the unit is ms.

Time sequence examination. For the convenience of description, the operation time of main protection, circuit breaker related to main protection, primary backup protection, circuit breaker related to primary backup protection, secondary backup protection and circuit breaker related to secondary backup protection is $\mathrm{T}_{1}-\mathrm{T}_{6}$. According to the occurring sequence of relay protection devices, priority level is formulated, namely the level of main protection is highest. Combined with the operation time, time sequence examination of alarm message can be carried out.

This paper uses the first alarm information as a benchmark at first, then check the other alarm information one by one to judge whether the information meet time constraints. If this alarm information exists, the first alarm information can be used as a reference to screen inconsistent time sequence information. On the other hand, if most information does not meet time constraints, the next information is adopted as a benchmark. Then, repeat the above process until find the reference information. The time constraint conditions of alarm information are shown in Table 1.

Table 1 Time constraint conditions

\begin{tabular}{cccccc}
\hline Time & $\mathrm{T}_{1}$ & $\mathrm{~T}_{2}$ & $\mathrm{~T}_{3}$ & $\mathrm{~T}_{4}$ & $\mathrm{~T}_{5}$ \\
\hline $\mathrm{T}_{1}$ & - & - & - & - & - \\
\hline $\mathrm{T}_{2}$ & {$[\mathbf{2 0 , 4 0 ]}$} & - & - & - & - \\
\hline $\mathrm{T}_{3}$ & {$[290,460]$} & {$[270,420]$} & - & - & - \\
\hline $\mathrm{T}_{4}$ & {$[310,500]$} & {$[290,460]$} & {$[20,40]$} & - & - \\
\hline $\mathrm{T}_{5}$ & {$[590,1060]$} & {$[570,1020]$} & {$[300,600]$} & {$[280,560]$} & - \\
\hline $\mathrm{T}_{6}$ & {$[610,1100]$} & {$[590,1060]$} & {$[320,640]$} & {$[300,600]$} & {$[20,40]$} \\
\hline
\end{tabular}

In Table $1,-$ means that the order of events does not meet time constraints, and the meaning of bold cell is that if the main protection operates at time $T_{1}$, the circuit breaker related to main protection must trip at time $\mathrm{T}_{2}$ after $20-40 \mathrm{~ms}$.

\section{Power system fault diagnosis based on TSFPN}

Process of fault diagnosis. In this paper, the process of power system fault diagnosis based on TSFPN is divided into the following steps:

1) Use the method of network topology analysis to search fault areas and acquire suspicious fault components;

2) Build the TSFPN models of suspicious fault components;

3) Check time sequence of alarm information and get rid of the inconsistent information, then set the initial confidence coefficient of all places; 
4) Utilize the TSFPN model to carry out fuzzy rational analysis and get the final confidence coefficients of suspicious fault components, then judge the real fault components and the operation situation of relay protection devices.

As is shown in Fig.4, IEEE 30-bus system is used to specify the modeling and reasoning process. In the system, $\mathrm{L}_{4-12 \mathrm{~m}}, \mathrm{~L}_{4-12 \mathrm{p}}, \mathrm{L}_{4-12 \mathrm{~s}}$ and $\mathrm{CB}_{4-12}$ represent the main protection, primary backup protection, secondary backup protection and circuit breaker of the side of line $\mathrm{L}_{4-12}$ which closes to bus $\mathrm{B}_{4} . \mathrm{L}_{12-4 \mathrm{~m}}, \mathrm{~L}_{12-4 \mathrm{p}}, \mathrm{L}_{12-4 \mathrm{~s}}$ and $\mathrm{CB}_{12-4}$ represent the main protection, primary backup protection, secondary backup protection and circuit breaker of the side of line $\mathrm{L}_{4-12}$ which closes to bus $\mathrm{B}_{12}$. $\mathrm{B}_{4 \mathrm{~m}}$ represents the main protection of bus $\mathrm{B}_{4}$.

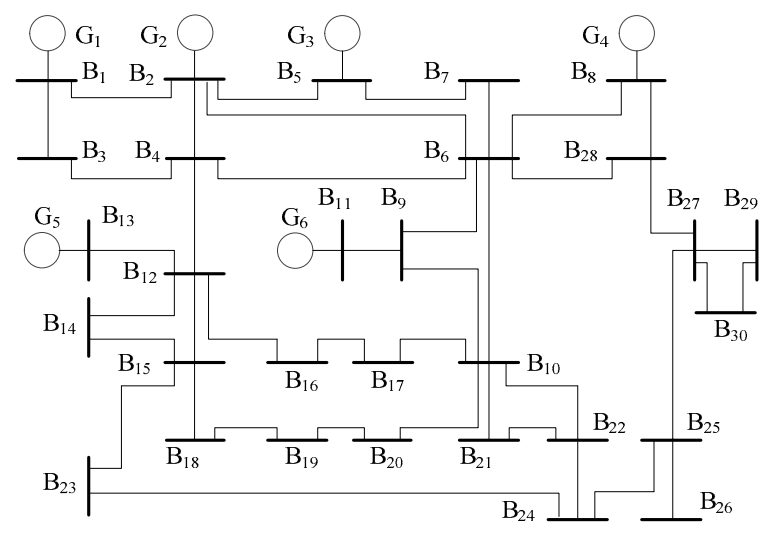

Fig.4 IEEE 30-bus system

Assume that the dispatch center has collected following alarm message: protection $\mathrm{B}_{24 \mathrm{~m}}(20 \mathrm{~ms})$ and $\mathrm{L}_{22-24 \mathrm{~s}}(750 \mathrm{~ms})$ operate, circuit breakers $\mathrm{CB}_{24-23}(50 \mathrm{~ms}), \mathrm{CB}_{24-25}(53 \mathrm{~ms})$ and $\mathrm{CB}_{22-24}$ (780ms) trip.

Up to now, the method of network topology analysis [13] has been widely used in quick search field, and the related theory has been relatively mature. Based on the above alarm information, this paper adopts the method of network topology analysis and the suspicious fault components are bus $\mathrm{B}_{24}$ and line $\mathrm{L}_{22-24}$.

TSFPN models. Take all expected alarm information related to component into consideration and combine fuzzy inference rules mentioned in above section, the TSFPN models of bus $\mathrm{B}_{24}$ and line $\mathrm{L}_{22-24}$ are shown in Fig.5 and Fig.6.

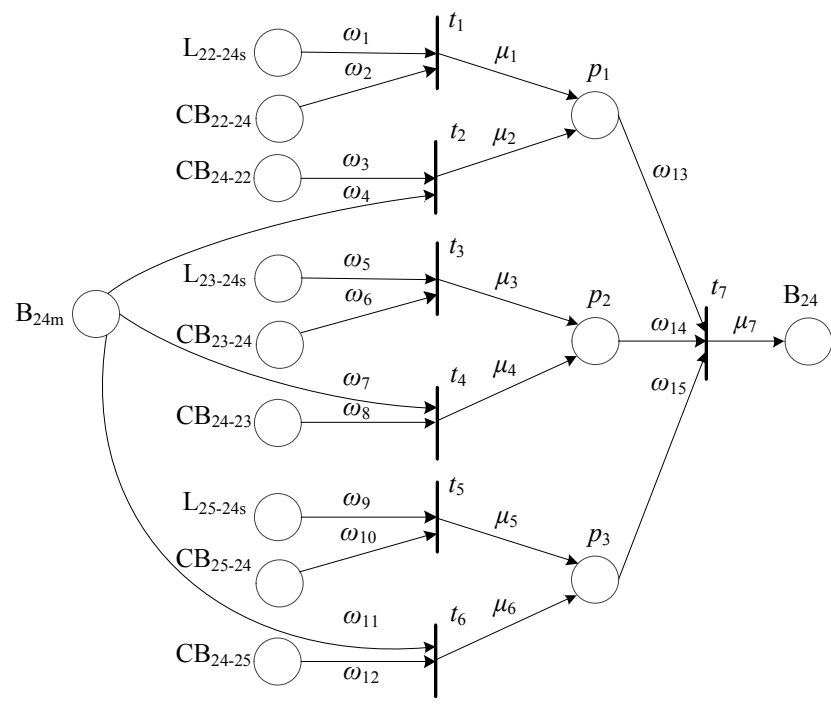

Fig.5 TSFPN model of bus $\mathrm{B}_{24}$

Alarm information screening. In this section, the time constraint conditions are used to examine time sequences of all alarm information. 
For bus $\mathrm{B}_{24}$, set the operation time of main protection $\mathrm{B}_{24 \mathrm{~m}}$ as a benchmark, so the time intervals between $\mathrm{CB}_{24-23}, \mathrm{CB}_{24-25}, \mathrm{~L}_{22-24 \mathrm{~s}}, \mathrm{CB}_{22-24}$ and $\mathrm{B}_{24 \mathrm{~m}}$ are $30 \mathrm{~ms}, 33 \mathrm{~ms}, 730 \mathrm{~ms}$ and $760 \mathrm{~ms}$. It is not hard to find that all alarm information meets time constraints according to Table 1.

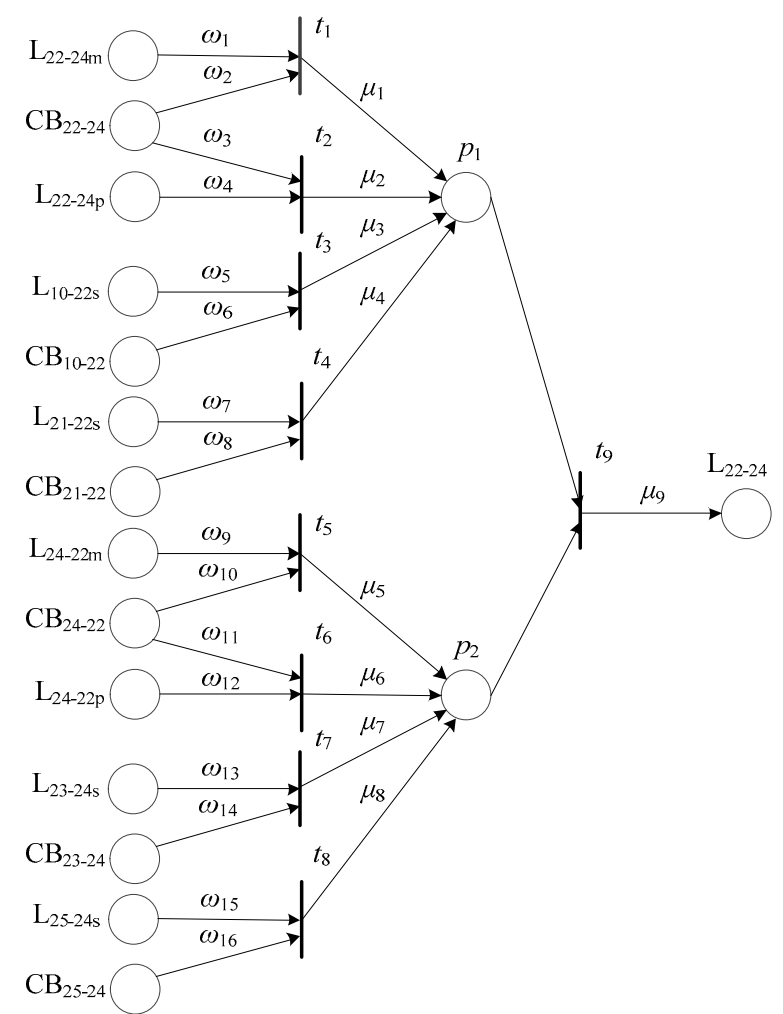

Fig.6 TSFPN model of line $\mathrm{L}_{22-24}$

According to the research achievements of literature [9], for the alarm information which meets time constraints, set the confidence coefficients of main protection, primary backup protection and secondary backup protection are $0.9,0.8$ and 0.7 , and the confidence coefficient of circuit breaker is 0.05 bigger than the related protection. On the other hand, for the other alarm information associated with component, set the confidence coefficients of protection and circuit breaker are 0.2.

Inference analysis. In order to improve the fault tolerance, the confidence coefficient of directed arc from transition to place is defined as 0.95 , and all directed arcs from place to transition have the same weight, and the sum is 1 [7].

On the basis of the above principles of parameter settings, fault diagnosis can be carried out by using the analysis method mentioned in above section. As an example, bus $\mathrm{B}_{24}$ is adopted to specify the process of fault diagnosis.

1) According to TSFPN model of bus $B_{24}$, matrices $\boldsymbol{I}, \boldsymbol{O}$ and initial confidence coefficient matrix $\boldsymbol{\theta}^{0}$ can be acquired

$$
\begin{aligned}
\boldsymbol{I} & =\left[\begin{array}{cccccccccccccc}
0.5 & 0.5 & 0 & 0 & 0 & 0 & 0 & 0 & 0 & 0 & 0 & 0 & 0 & 0 \\
0 & 0 & 0.5 & 0.5 & 0 & 0 & 0 & 0 & 0 & 0 & 0 & 0 & 0 & 0 \\
0 & 0 & 0 & 0 & 0.5 & 0.5 & 0 & 0 & 0 & 0 & 0 & 0 & 0 & 0 \\
0 & 0 & 0 & 0.5 & 0 & 0 & 0.5 & 0 & 0 & 0 & 0 & 0 & 0 & 0 \\
0 & 0 & 0 & 0 & 0 & 0 & 0 & 0.5 & 0.5 & 0 & 0 & 0 & 0 & 0 \\
0 & 0 & 0 & 0.5 & 0 & 0 & 0 & 0 & 0 & 0.5 & 0 & 0 & 0 & 0 \\
0 & 0 & 0 & 0 & 0 & 0 & 0 & 0 & 0 & 0 & 0.33 & 0.33 & 0.33 & 0
\end{array}\right]^{\mathrm{T}} \\
\boldsymbol{O} & =\left[\begin{array}{llllllllllllll}
0 & 0 & 0 & 0 & 0 & 0 & 0 & 0 & 0 & 0 & 0.95 & 0 & 0 & 0 \\
0 & 0 & 0 & 0 & 0 & 0 & 0 & 0 & 0 & 0 & 0.95 & 0 & 0 & 0 \\
0 & 0 & 0 & 0 & 0 & 0 & 0 & 0 & 0 & 0 & 0 & 0.95 & 0 & 0 \\
0 & 0 & 0 & 0 & 0 & 0 & 0 & 0 & 0 & 0 & 0 & 0.95 & 0 & 0 \\
0 & 0 & 0 & 0 & 0 & 0 & 0 & 0 & 0 & 0 & 0 & 0 & 0.95 & 0 \\
0 & 0 & 0 & 0 & 0 & 0 & 0 & 0 & 0 & 0 & 0 & 0 & 0.95 & 0 \\
0 & 0 & 0 & 0 & 0 & 0 & 0 & 0 & 0 & 0 & 0 & 0 & 0 & 0.95
\end{array}\right]
\end{aligned}
$$




$$
\boldsymbol{\theta}^{0}=[0.7,0.75,0.2,0.9,0.2,0.2,0.95,0.2,0.2,0.95,0,0,0,0]
$$

2) Calculate the next state of places by using Eq. 2 and Eq. 3, then get $\boldsymbol{\theta}^{1}=[0,0,0,0,0,0,0,0,0$, $0,0.6887,0.8788,0.8788,0]$. At this time the confidence coefficients of middle places can be acquired, then do the calculation again, and get $\boldsymbol{\theta}^{2}=[0,0,0,0,0,0,0,0,0,0,0,0,0,0.7669]$.

3) Because $\boldsymbol{\theta}^{2}$ only has one nonzero element, so the inference analysis is over, and the confidence coefficient of terminal place, namely the fault probability of bus $\mathrm{B}_{24}$ is 0.7669 .

Similarly, the fault probability of line $\mathrm{L}_{22-24}$ is 0.3497 . This paper fully takes the uncertainty of alarm information into consideration, and defines that if the confidence coefficient of terminal place is greater than 0.65 , the corresponding component is faulted. Based on this, the fault component is bus $\mathrm{B}_{24}$. Furthermore, the circuit breaker $\mathrm{CB}_{24-22}$ refuses to trip, so secondary backup protection $\mathrm{L}_{22-24 \mathrm{~s}}$ operates and triggers circuit breaker $\mathrm{CB}_{22-24}$ after a certain time delay.

\section{Case study}

To better demonstrate the effectiveness of the fault diagnosis method proposed in this paper, IEEE 30-bus power system is still adopted. Three simulation examples are carried out, and the fault diagnosis results are shown as follows.

1) Simple fault diagnosis with complete information is studied. Suppose that protection $B_{23 \mathrm{~m}}$ (15ms) operates, circuit breakers $\mathrm{CB}_{23-15}(41 \mathrm{~ms})$ and $\mathrm{CB}_{23-24}(43 \mathrm{~ms})$ trip.

Through the analysis of network topology, the suspicious fault component is bus $\mathrm{B}_{23}$. In addition, all alarm information meet time constraints, so by fuzzy reasoning, the confidence coefficient of bus $\mathrm{B}_{23}$ is 0.8348 and bigger than 0.65 , which shows that bus $\mathrm{B}_{23}$ is really faulted, furthermore, protective relays and circuit breakers operate rightly.

2) Simple fault diagnosis with uncertain alarm information is studied. Suppose that protection $\mathrm{L}_{19-20 \mathrm{~m}}(23 \mathrm{~ms}), \mathrm{L}_{20-19 \mathrm{~m}}(24 \mathrm{~ms}), \mathrm{L}_{19-20 \mathrm{p}}(340 \mathrm{~ms})$ and $\mathrm{L}_{18-19 \mathrm{~s}}(670 \mathrm{~ms})$ operate, circuit breakers $\mathrm{CB}_{20-19}(51 \mathrm{~ms}), \mathrm{CB}_{19-20}(90 \mathrm{~ms})$ and $\mathrm{CB}_{18-19}(695 \mathrm{~ms})$ trip.

In this scenario, the suspicious fault components are line $\mathrm{L}_{18-19}$ and line $\mathrm{L}_{19-20}$. By checking the actual alarm information related to each suspicious component, only the information " $\mathrm{CB}_{19-20}(90 \mathrm{~ms})$ trips" does not meet time constraint. Then, the remaining information is used for fuzzy reasoning and the confidence coefficients of suspicious fault components are 0.4738 and 0.7446 , so the actual fault component is line $\mathrm{L}_{19-20}$. Through further analysis of actual alarm information, it is not hard to find that circuit breaker $\mathrm{CB}_{19-20}$ refuses to trip. In addition, if this article does not introduce time sequence examination, it may get the wrong conclusion that $\mathrm{L}_{18-19 \mathrm{~s}}$ and $\mathrm{CB}_{18-19}$ mal-operate.

3) Complex fault diagnosis with uncertain and incomplete alarm information is studied. Suppose that protection $\mathrm{L}_{10-21 \mathrm{~m}}(27 \mathrm{~ms}), \mathrm{L}_{21-10 \mathrm{~m}}(28 \mathrm{~ms}), \mathrm{B}_{22 \mathrm{~m}}(30 \mathrm{~ms})$ and $\mathrm{L}_{21-10 \mathrm{p}}(130 \mathrm{~ms})$ operate, circuit breakers $\mathrm{CB}_{22-24}(53 \mathrm{~ms}), \mathrm{CB}_{22-10}(54 \mathrm{~ms}), \mathrm{CB}_{21-10}(59 \mathrm{~ms}), \mathrm{CB}_{10-21}(60 \mathrm{~ms})$ and $\mathrm{CB}_{21-22}(683 \mathrm{~ms})$ trip.

The suspicious fault components are line $\mathrm{L}_{10-21}$, bus $\mathrm{B}_{21}$, line $\mathrm{L}_{21-22}$ and bus $\mathrm{B}_{22}$. By checking the actual alarm information, the information " $\mathrm{L}_{21-10 \mathrm{p}}(130 \mathrm{~ms})$ operates" related to line $\mathrm{L}_{10-21}$ does not meet time constraint. Then by calculation, the confidence coefficients of all suspicious fault components are $0.8348,0.5189,0.3497$ and 0.7148 , so the result is that line $\mathrm{L}_{10-21}$ and bus $\mathrm{B}_{22}$ are all faulted. In addition, after main protection $\mathrm{B}_{22 \mathrm{~m}}$ operates, circuit breaker $\mathrm{CB}_{22-21}$ refuses to trip, so secondary backup protection operates and triggers the corresponding circuit breaker. But only circuit breaker $\mathrm{CB}_{21-22}$ trips at $683 \mathrm{~ms}$, and this information meets time constraint, so the information of secondary backup protection $\mathrm{L}_{21-22 \mathrm{~s}}$ is missing.

The above simulation examples show that the fault diagnosis model and reasoning method can accurately judge the fault components and have a good application prospect. 


\section{Summary}

This paper proposes a power system fault diagnosis method based on TSFPN which not only takes the influence of incomplete and uncertain alarm information on fault diagnosis into consideration, but also utilizes the time constraint characteristics of protective relays and circuit breakers to remove inconsistent time sequence information. Simulation results on IEEE 30-bus power system indicate that this method has high fault tolerance and can obtain satisfying results in the situation with complete, uncertain, inconsistent and missing information.

\section{References}

[1] Zhao Wei, Bai Xiaomin, Ding Jian, et al. A new fault diagnosis approach of power grid based on cooperative expert system and multi-agent technology[J]. Proceedings of the CSEE, 2006, 26(20): $1-8$.

[2] Liu Chao, He Zhengyou, Yang Jianwei. A quantum neural network based fault diagnosis algorithm for power grid[J]. Power System Technology, 2008, 32(9): 56-60.

[3] Zhang Zhiyi, Yuan Rongxiang, Yang Tongzhong, et al. Rule extraction for power system fault diagnosis based on the combination of rough sets and niche genetic algorithm[J]. Transactions of China Electrotechnical Society, 2009, 24(1):158-163.

[4] Song Gongyi, Wang Xiaoru, Zhou Shu. Research on complex faults diagnosis of multi-area power network based on Bayesian networks[J]. Power System Protection and Control, 2011,39(7): 20-25.

[5] Xiong Guojiang, Shi Dongyuan. Improved fault-tolerant Petri nets for fault-diagnosis power grids[J]. Journal of Huazhong University of Science and Technology: Natural Science Edition, 2013, 41(1): 11-15.

[6] Sun Jing, Qin Shiyin, Song Yonghua. Fault diagnosis of electric power systems based on fuzzy Petri nets[J]. IEEE Trans on Power Systems, 2004, 19(4): 2053-2059.

[7] Yang Jianwei, He Zhengyou, Zang Tianlei. Power system fault -diagnosis method based on directional weighted fuzzy petri nets[J]. Proceedings of the CSEE, 2010, 30(34): 42-49.

[8] Xie Hongtao, Tong Xiaoyang. A method of synthetical fault diagnosis for power system based on fuzzy hierarchical Petri net[J]. Power System Technology, 2012, 36(1): 246-252.

[9] Yang Jianwei, He Zhengyou. Power system fault diagnosis approach based on time sequence fuzzy petri net[J]. Automation of Electric Power Systems, 2011, 35(15): 46-51.

[10] Tong Xiaoyang, Xie Hongtao, Sun Mingwei. Power system fault diagnosis model based on layered fuzzy petri net considering temporal constraint checking[J]. Automation of Electric Power Systems, 2013, 37(6): 63-68.

[11] Wu Wenke, Wen Fushuan, Xue Yusheng, et al. A weighted fuzzy petri net based model with time-delay constraints for power system fault diagnosis employing information from multiple sources[J]. Automation of Electric Power Systems, 2013, 37(24): 43-53.

[12]Kang Taifeng, Wu Wenchuan, Zhang Boming, et al. Temporal abductive reasoning based diagnosis and alarm for power grid[J]. Proceedings of the CSEE, 2010, 30(19): 84-90.

[13]Zhang Lin. The fault diagnosis of electric power systems based on fuzzy Petri nets[D]. Jiangxi: Nanchang University, 2007. 